\title{
Neuroimmune signaling in neuropsychiatric disease
}

\section{A. Leslie Morrow ${ }^{1}$ - Lawrence H. Price ${ }^{2}$}

Published online 21 October 2019

(C) Springer-Verlag GmbH Germany, part of Springer Nature 2019

It is our pleasure to introduce this Special Issue of Psychopharmacology focused on "Neuroimmune Signaling in Neuropsychiatric Disease". This issue contains recent work from established and emerging thought leaders in the field that contributes to our understanding of the role of neuroimmune signaling. We thank them for their efforts and contributions to the journal.

The innate immune system is implicated and activated in virtually every neuropsychiatric condition, but our understanding of the role of these processes in disease is poorly understood. Neuroimmune signaling can involve both proinflammatory and anti-inflammatory processes that in turn can alter neurotransmission at multiple sites. This represents a new frontier in our understanding of normal and pathological brain function, as well as the relationship between system- ic and CNS disease. We believe this Special Issue will contribute to the important insights that the study of neuroimmune signaling can afford us into the etiology of CNS disease and the identification of new targets for treatment.

We hope you enjoy the contributions in this issue and that they help inspire you to address neuroimmune signaling in your research.

Best regards,

A. Leslie Morrow and Lawrence H. Price

Publisher's note Springer Nature remains neutral with regard to jurisdictional claims in published maps and institutional affiliations.

This article belongs to a Special Issue on Neuroimmune Signaling in Psychiatric Disease.

A. Leslie Morrow

morrow@med.unc.edu

1 Departments of Psychiatry and Pharmacology, University of North Carolina School of Medicine, 3027 Thurston-Bowles Building, CB 7178, Chapel Hill, NC 27599-7178, USA

2 Brown University, Warren Alpert Medical School, Butler Hospital, 345 Blackstone Blvd, Providence, RI 02906, USA 